\title{
Pequenas espécies de Parmeliaceae ciliadas no Parque Estadual da Cantareira, São Paulo, SP, Brasil: gêneros Bulbothrix, Parmelinella e Parmelinopsis (Parmeliaceae, Ascomycota)
}

\author{
Michel Navarro Benatti ${ }^{1,2}$
}

Recebido: 14.02.2012; aceito: 26.04 .2012

\begin{abstract}
Small ciliate Parmeliaceae species in Parque Estadual da Cantareira, São Paulo, São Paulo State, Brazil: the genera Bulbothrix, Parmelinella, and Parmelinopsis (Parmeliaceae, Ascomycota)). The survey of species belonging to the genera of small ciliate parmeliae in Parque Estadual da Cantareira and surrounding areas revealed the occurrence of two Bulbothrix, one Parmelinella, and four Parmelinopsis species. The species are characterized by foliose thalli with lobes or laciniae, generally $\leq 0.5 \mathrm{~cm}$ wide. Bulbothrix has marginal bulbate cilia, Parmelinella simple axillary cilia, and Parmelinopsis simple conspicuous cilia. Medulary chemical constituents are very diverse in Bulbothrix and Parmelinopsis, while salazinic acid predominates in Parmelinella. An identification key, descriptions, comments, and illustrations are provided.

Key words: Cantareira Mountain Range, cilia, lichenized fungi
\end{abstract}

RESUMO - (Pequenas espécies de Parmeliaceae ciliadas no Parque Estadual da Cantareira, São Paulo, SP, Brasil: gêneros Bulbothrix, Parmelinella e Parmelinopsis (Parmeliaceae, Ascomycota)). O levantamento das espécies pertencentes aos gêneros de pequenas parmélias ciliadas no Parque Estadual da Cantareira e arredores revelou a ocorrência de duas espécies de Bulbothrix, uma de Parmelinella e quatro de Parmelinopsis. As espécies são caracterizadas pelos talos foliosos com lobos ou lacínias em geral $\leq 0,5 \mathrm{~cm}$ larg., sendo que Bulbothrix apresenta cílios marginais bulbados, Parmelinella apresenta cílios simples axilares e Parmelinopsis apresenta cílios simples conspícuos. Constituintes químicos medulares são muito diversos em Bulbothrix e Parmelinopsis, enquanto que o ácido salazínico é predominante em Parmelinella. São apresentados uma chave de identificação, descrições, comentários e ilustrações.

Palavras-chave: cílios, fungos liquenizados, Serra da Cantareira

\section{Introdução}

O Parque Estadual da Cantareira está situado ao norte do município de São Paulo, entre as latitudes $23^{\circ} 20^{\prime}$ e $23^{\circ} 27^{\prime} \mathrm{S}$ e as longitudes $46^{\circ} 28^{\prime}$ e $46^{\circ} 42^{\prime} \mathrm{W}$, ocupando parte do Maciço da Cantareira e parte da Serra de Pirucaia, entre as Serras do Mar, de Paranapiacaba e da Mantiqueira (Soares 1999). A temperatura média anual é de $18,2{ }^{\circ} \mathrm{C}$, a média de pluviosidade anual é de $1.400 \mathrm{~mm}$ e a altitude varia entre 750 a 1.215 m (Soares 1999).

A Reserva conta com uma área estimada em 7.900 ha distribuídos pelos municípios de São Paulo, Caieiras, Mairiporã e Guarulhos. Está atualmente sob a jurisdição do Instituto Florestal, sob efeito do artigo $2^{\circ}$ do Código Florestal Brasileiro. A data efetiva da criação do Parque Estadual é de 30 de Janeiro de 1963, conforme o decreto estadual 41.626 (São Paulo 1988).

A Reserva é praticamente coberta por floresta, em sua maior parte de Mata Atlântica (floresta ombrófila densa e floresta estacional semidecídua), bem caracterizada devido à posição geográfica, na porção sul da Serra da Mantiqueira. Os diferentes fatores geomorfológicos somados ao fator de interferência humana agem setorizando a vegetação e também abrindo pequenas clareiras. Acrescenta-se a isso as áreas de reflorestamento experimental do Instituto Florestal e remanescentes dos antigos sítios (São Paulo 1988).

Dados aproximados do número de espécies de fungos liquenizados para o Brasil indicam para a Mata Atlântica um total de 400 espécies publicadas

1. Instituto de Botânica, Núcleo de Pesquisa em Micologia, Caixa Postal 68041, 04045-972 São Paulo, SP, Brasil

2. Autor para correspondência: michel_benatti@yahoo.com.br 
em uma estimativa total de 850 espécies esperadas para a região na qual está inclusa a Serra da Cantareira (Marcelli 1998a). Para a região da Serra da Cantareira, até o presente conhece-se apenas uma relação de 111 espécies de fungos liquenizados pertencentes a 47 gêneros (Marcelli 1998b), sem maiores detalhes sobre as espécies.

Este trabalho objetivou realizar o levantamento das espécies foliosas de Bulbothrix Hale, Parmelinella Elix \& Hale e Parmelinopsis Elix \& Hale que ocorrem no Parque Estadual da Cantareira e regiões circunvizinhas. São fornecidos uma chave artificial de identificação, descrições comentadas, dados das localidades e de ambientes para as espécies encontradas.

\section{Material e métodos}

A metodologia de coleta para fungos liquenizados foliosos é a descrita em Hale (1987), Malcolm \& Galloway (1997) e Benatti \& Marcelli (2007). As análises morfológicas consistiram na observação das características macro e microscópicas dos espécimes, por meio de estereomicroscópio e microscópio óptico. Foram analisadas as estruturas somáticas e de reprodução, tanto direta como indireta dos talos.

O material coletado na área da Reserva da Serra da Cantareira e depositado no herbário Maria Eneyda P. Kauffmann Fidalgo (SP) do Instituto de Botânica foi identificado conforme a metodologia tradicional de liquenologia, descrita em Hale (1979), Galloway (1985, 2007) e Fink (1905).

A metodologia de análises químicas consistiu em testes de coloração, irradiação por luz UV, testes de microcristalização com reagentes G.E. e G.A.W., e cromatografia em camada delgada (CCD) com solvente C, seguindo Asahina \& Shibata (1954), Walker \& James (1980), White \& James (1985), Huneck \& Yoshimura (1996), Bungartz (2001) e Orange et al. (2001).

\section{Resultados e discussão}

Foram analisadas 52 amostras de fungos liquenizados foliosos pertencentes aos gêneros Bulbothrix, Parmelinella e Parmelinopsis da família Parmeliaceae. No Parque Estadual da Cantareira foram encontradas sete espécies de pequenas parmélias ciliadas. Embora todas sejam conhecidas para o Estado, existem poucas citações (vide distribuições geográficas das espécies) e este é o primeiro registro para a localidade de estudo, densa área florestal em meio a maior região urbana do continente sul americano, e o primeiro registro em reserva de Mata Atlântica em meio urbano no Brasil.

As duas espécies de Bulbothrix e a única espécie de Parmelinella encontradas apresentam ácidos norstíctico e salazínico (reações medulares $\mathrm{K}+$ amarelo $\rightarrow$ alaranjado ou vermelho, $\mathrm{P}+$ amarelo ou alaranjado), enquanto que as espécies de Parmelinopsis apresentam em geral substâncias do "complexo horrescens" e quantidades variáveis de ácido girofórico (reações medulares $\mathrm{C}+$ róseo ou avermelhado e KC-, ou $\mathrm{C}+\mathrm{e} \mathrm{KC}+$ róseos ou avermelhados).

Bulbothrix ventricosa (Hale \& Kurokawa) Hale, B. tabacina (Nylander) Hale, Parmelinella cinerascens (Lynge) Benatti \& Marcelli, Parmelinopsis horrescens (Taylor) Elix \& Hale e P. minarum (Vainio) Elix \& Hale apresentam formação de isídios, enquanto que P. schindleri (Hale) Elix \& Hale apresenta formação de lacínulas e P. subfatiscens (Kurokawa) Elix \& Hale formação de pústulas. Todos os espécimes encontrados são corticícolas em troncos, galhos ou ramos de árvores; algumas espécies como $P$. minarum e $P$. schindleri estão com frequência crescendo entrelaçadas com talos de briófitas (musgos e hepáticas). Nenhuma espécie saxícola foi encontrada.

Poucos espécimes apresentaram formação de apotécios e, mesmo esses, somente com himênios pouco desenvolvidos e no máximo ascos imaturos. Embora alguns espécimes tenham apresentado picnídios, mesmo em grande quantidade como no caso de $P$. schindleri, não foram encontrados conídios nos materiais.

Chave de identificação para as espécies de pequenas parmélias ciliadas do Parque Estadual da Cantareira

1. Cílios bulbados

2. Bulbos ciliares laminais comuns; lado de baixo com mesclas de negro e/ou marrom; apotécios

(quando presentes) coronados; medula com ácido norestíctico .................................... Bulbothrix ventricosa

2. Bulbos ciliares laminais ausentes; lado de baixo negro com margens marrons; apotécios (quando

presentes) ecoronados; medula com ácido salazínico ..................................................... Bulbothrix tabacina

1. Cílios não bulbados

3. Cílios escassos, normalmente axilares (curtos $\leq 0,5 \mathrm{~mm}$ compr.); medula com ácido salazínico 
3. Cílios frequentes a abundantes, por toda a margem; medula com outros ácidos

4. Talo com lacínulas laminais ciliadas

Parmelinopsis schindleri

4. Talo com isídios ou pústulas

5. Talo pustulado, pústulas ciliadas e eventualmente rompendo (sem originar sorédios)

5. Talo isidiado

6. Isídios eciliados, medula $\mathrm{C}+\mathrm{e} \mathrm{KC}+$ róseos (ácido girofórico)

Parmelinopsis subfatiscens

6. Isídios ciliados, medula C-, $\mathrm{KC}+$ róseo (substâncias do "complexo horrescens"

às vezes com traços de ácido girofórico) Parmelinopsis horrescens

Bulbothrix tabacina (Montagne \& Bosch) Hale, Phytologia 28: 481. 1974. 三 Parmelia tabacina Montagne \& Bosch. Sylloge generum specierumque cryptogamarum: 327. 1856. Tipo: INDONÉSIA. JAVA: in cortice arborum, leg. Junghuhn s.n. (lectótipo L!, duplicata em PC!). Ilustrações em Hale (1976b) e Marcelli (1993).

Talo pardo esverdeado claro em herbário, com até $2,5 \mathrm{~cm}$ diâm. Lacínias de ramificação subdicotômica a irregular, (0,5-)1,5-3,0 mm larg., pouco adnatos, pouco aderidos, contíguos a raramente sobrepostos lateralmente, de ápices subarredondados a subtruncados, a margem sinuosa, crenada a subirregular com linha negra sutil, as axilas ovais, a superfície contínua com raras quebras irregulares, lisa a subrugosa, sem formação de bulbos ciliares. Lacínulas ou lóbulos adventícios ausentes. Cílios negros, simples de ápices longos e às vezes curvos, frequentes, $0,10-0,25 \times$ ca. $0,03 \mathrm{~mm}$, bulbados, bulbos 0,05-0,15 mm larg., contíguos nas axilas e solitários nas crenas dos lobos. Máculas fracas, puntiformes, laminais. Pseudocifelas ausentes. Sorédios e pústulas ausentes. Isídios 0,05-0,15 × ca. 0,05 mm, granulares a cilíndricos lisos, simples em geral, laminais (escassos e em pequenos grupos), eretos, firmes a caducos, concoloridos, sem ornamentações. Medula branca, sem manchas de pigmentos $\mathrm{K}+$ púrpura. Lado de baixo negro, lustroso, liso a subrugoso, zona marginal negra a marrom escura, indistinta a atenuada, lustrosa, lisa a subrugosa, papilada, parcialmente rizinada. Rizinas $0,10-0,30 \times$ ca. $0,05 \mathrm{~mm}$, negras, às vezes marrons escuras próximas às margens, de bases não bulbadas, simples, homogeneamente distribuídas, frequentes. Apotécios e picnídios não encontrados.

Substâncias de importância taxonômica: atranorina e cloroatranorina (córtex superior, $\mathrm{K}+\mathrm{am}, \mathrm{UV}-$ ), ácidos salazínico e consalazínico (medula, $\mathrm{K}+$ amarelo $\rightarrow$ vermelho, C-, KC-, P+ alaranjado, UV-).
Distribuição conhecida: Oceania, Ásia, África, América do Norte, Caribe, América do Sul. No Brasil é citada para os Estados de RS e SP (Junghuhn 1855, Montagne 1856, Zahlbruckner 1928, Dodge 1959, Awasthi 1976, Hale 1976b, López-Figueiras 1986, Swinscow \& Krog 1988, Osorio 1989, 1992, Aptroot 1990, Marcelli 1990, 1991, 1993, Kurokawa 1993, Elix 1994a, Marcano et al. 1996, Galloway \& Quilhot 1998, Sipman \& Wolf 1998, Louwhoff \& Elix 2000, 2002, Kurokawa \& Lai 2001, Canêz 2005, Divakar \& Upreti 2005, Jungbluth 2006, Feuerer 2008, Benatti 2010).

Material estudado: BRASIL. SÃo PAULo: Município de São Paulo, Serra da Cantareira, Parque Estadual da Cantareira, VI-2000, leg. M.N. Benatti 1410 (SP).

Comentários: Bulbothrix tabacina é caracterizada pela presença de isídios simples laminais, pelos cílios simples bulbados axilares ou em crenas, pelo córtex inferior de coloração negra e pela constituição química medular (ácido salazínico).

O único espécime encontrado está sobre ramo fino e trata-se de um talo pequeno e pouco desenvolvido, sem ascomas ou picnídios. Não há muitos isídios no material, embora haja várias marcas deixadas pela queda dessas estruturas sobre o córtex. Diferente de $B$. ventricosa, essa espécie nunca apresenta formação de bulbos laminais; a coloração do córtex inferior é sempre predominatemente negra, e os apotécios, quando presentes, diferem nas duas espécies sendo ecoronados em B. tabacina e coronados em B. ventricosa (Benatti 2010).

Hale (1976b) mencionou que esta é a espécie isidiada do gênero mais comumente coletada. Pode ser distinguida de $B$. isidiza que possui coloração marrom muito clara no córtex inferior, em contraste ao negro de margens marrons escuras de B. tabacina. Conforme Hale (1976b) e Benatti (2010), B. isidiza possui o talo mais fino e delicado em comparação, mais para membranáceo. 
Bulbothrix ventricosa (Hale \& Kurokawa) Hale, Phytologia 28 (5): 481.1974 E Parmelia isidiza var. domingensis Vainio. Annales Academiae Scientarum Fennicae 6A(7): 17. 1915. Tipo: REPÚBLICA DOMINICANA, SANTo Domingo, La Cumbra, ad corticem arboris, 09-IV-1906, leg. C. Raunkiaer 492, (lectótipo TUR-V!, duplicata em C n.v.).

Ilustrações em Hale (1976b) e Benatti (2012b).

Talo pardo esverdeado (às vezes claro) em herbário, com até $7,0 \mathrm{~cm}$ diâm. Lobos de ramificação subdicotômica a irregular, 1,0-3,0(-4,0) mm larg., adnatos, aderidos, contíguos a pouco sobrepostos lateralmente, de ápices subarredondados a subtruncados, a margem crenada a subirregular sem linha negra, as axilas ovais, a superfície contínua, lisa a subrugosa, frequentemente com bulbos ciliares, escassos a abundantes. Lacínulas ou lóbulos adventícios ausentes. Cílios negros, simples ou sem ápices, frequentes, $0,10-0,30 \times$ ca. $0,03 \mathrm{~mm}$, bulbados, bulbos $0,05-0,30 \mathrm{~mm}$ larg., contíguos nas axilas e solitários nas crenas dos lobos. Máculas fracas a distintas, puntiformes, laminais. Pseudocifelas ausentes. Sorédios e pústulas ausentes. Isídios 0,05-0,20 × ca. $0,05 \mathrm{~mm}$, granulares a cilíndricos lisos, simples em geral a raramente pouco ramificados, laminais, eretos, firmes às vezes caducos, concoloridos ou de ápices amarronzados, sem ornamentações. Medula branca, sem manchas de pigmentos $\mathrm{K}+$ púrpura. Lado de baixo mesclado, marrom claro a escuro ou negro, lustroso, liso a rugoso ou parcialmente venado, zona marginal marrom clara a escura indistinta do centro, lustrosa, lisa, papilada, parcialmente rizinada. Rizinas $0,10-0,30 \times$ ca. $0,05 \mathrm{~mm}$, marrons, às vezes de ápices claros, de bases parcialmente bulbadas escuras, simples, homogeneamente distribuídas, frequentes. Apotécios e picnídios não encontrados.

Substâncias de importância taxonômica: atranorina e cloroatranorina (córtex superior, $\mathrm{K}+$ amarelo, UV-), ácidos norstíctico e conorstíctico (medula, $\mathrm{K}+$ amarelo $\rightarrow$ alaranjado ou vermelho claro, C-, KC-, P+lr, UV-).

Distribuição conhecida: Ásia, África, América do Norte, América Central, Caribe e América do Sul. No Brasil é citada para os Estados de MG, SC, SP, PA e PR (Vainio 1915, Hale 1976b, Hale \& Kurokawa 1964, Brako et al. 1985, López-Figueiras 1986, Swinscow \& Krog 1988, Marcano et al. 1996, Eliasaro \& Adler 1997, Ribeiro 1998, Eliasaro 2001, Jungbluth
2006, Papong et al. 2007, Benatti 2010, Gumboski \& Eliasaro 2011).

Material estudado: BRASIL. SÃo PAULo: Município de São Paulo, Serra da Cantareira, Parque Estadual da Cantareira, VI-2000, leg. M.N. Benatti 1042, 1301, 1303, 1390, 1397 (SP); idem, Núcleo da Pedra Grande, 18-V-1992, leg. M.P. Marcelli, A. Rezende \& O. Yano 13607 (SP).

Comentários: Bulbothrix ventricosa é caracterizada pela presença de isídios simples e laminais, pelos cílios simples, bulbados, axilares ou em crenas, pelo córtex inferior de coloração mesclada variável e pela constituição química medular (ácido norstíctico).

Esta espécie é aparentemente comum sobre ramos na localidade de estudo, possivelmente pelo ambiente em geral mais fechado e escuro que dificulta seu estabelecimento sobre os troncos pouco iluminados. Há em todos os espécimes a presença frequente de bulbos ciliares negros, brilhantes e semi-imersos encontrados por toda a lâmina, embora sejam escassos em alguns talos e muito comuns em outros.

Esta espécie é similar a Bulbothrix tabacina, sendo que ambas tem como características a presença de isídios laminais. Porém, B. tabacina contém ácido salazínico como principal constituinte medular ao invés do ácido norstíctico, e os ápices dos cílios também são mais nítidos e alongados (até duplos ocasionalmente) em comparação direta, vertendo para baixo muitas vezes auxiliando na fixação do talo ao substrato. Além disso, B. tabacina tem o córtex inferior negro com margem marrom atenuada, bem delimitado.

Parmelinella cinerascens (Lynge) Benatti \& Marcelli, Opuscula Philolichenum 11:27. $2012 \equiv$ Parmelia cinerascens Lynge, Arkiv för Botanik 13: 104. 1914. Tipo: PARAGUAI. Paraguari, 02/VIII/1893, ad arborem sat solitariam, in ripa rivuli campi, G.O. Malme 1498 (holótipo S!, epítipo SP!, isoepítipo S!). Ilustrações em Benatti (2012a).

Talo cinza esverdeado claro a pardo esverdeado em herbário, lobado, com até $10,0 \mathrm{~cm}$ diâm. Lobos de ramificação irregular a subdicotômica, 1,0-3,5(-5,0) mm larg., adnatos, aderidos, contíguos a sobrepostos lateralmente ou raramente amontoados, de ápices arredondados, a margem lisa a subcrenada com linha negra parcial, as axilas ovais, a superfície contínua e lisa tornando-se pouco rugosa no centro, às vezes com quebras irregulares. Lacínulas ausentes, lóbulos adventícios ausentes a ocasionais em 
partes velhas. Cílios negros, simples, escassos, $0,10-0,30 \times$ ca. $0,03 \mathrm{~mm}$, restritos na maioria às axilas ou crenas dos lobos. Máculas fracas a ausentes, puntiformes, laminais. Pseudocifelas ausentes. Pústulas e sorédios ausentes. Isídios $0,10-0,30(-0,40) \times$ ca. $0,05 \mathrm{~mm}$, cilíndricos, lisos, em geral simples a raramente pouco ramificados, laminais, eretos, firmes a caducos, concoloridos ou de ápices marrons a negros, sem ornamentações. Medula branca, sem manchas de pigmentos $\mathrm{K}+$ púrpura. Lado de baixo negro, lustroso, de rugoso a venado, papilado, zona marginal marrom clara a escura, atenuada, lustrosa, pouco rugosa a venada, tornando-se papilada e rizinada na transição para o centro. Rizinas $0,10-0,50(-0,60) \times$ ca. $0,05 \mathrm{~mm}$, marrons escuras a negras, simples, frequentes a abundantes, homogeneamente distribuídas, porém, às vezes parcialmente aglomeradas. Apotécios côncavos a cupuliformes, 0,5-2,0 mm diâm., sésseis a subestipiados, imperfurados, margem lisa a isidiada, anfitécio rugoso a isidiado, disco marrom, não pruinoso. Ascósporos não encontrados (somente ascos imaturos). Picnídios não encontrados.

Substâncias de importância taxonômica: atranorina e cloroatranorina (córtex superior, $\mathrm{K}+$ amarelo, UV-), ácidos salazínico e consalazínico (medula, $\mathrm{K}+$ amarelo $\rightarrow$ vermelho escuro, C-, KC-, $\mathrm{P}+$ amarelo, UV-).

Distribuição conhecida: Ásia e América do Sul. No Brasil é citada para os Estados de MG, SP e PE (Lynge 1914, Hale 1976a, Ribeiro 1998, Marcelli 2004, Divakar \& Upreti 2005, Jungbluth 2006, Feuerer 2008, Benatti 2012a).

Material estudado: BRASIL. São PaUlo: Município de São Paulo, Serra da Cantareira, Parque Estadual da Cantareira, sobre tronco de árvore na mata, VI-2000, leg. M.N. Benatti 1400 (SP); idem, na beira da estrada do Pé de galinha para Mairiporã, 25-I-1991, leg. M.P. Marcelli, A. Rezende \& I. Riquelme 11584 (SP).

Comentários: Parmelinella cinerascens é caracterizada pela presença de isídios simples laminais, pelos esparsos e curtos cílios axilares, pelo córtex inferior negro rugoso de margens marrons e pela constituição química medular (ácido salazínico).

Pode ser diferenciada das demais espécies de pequenas parmélias ciliadas encontradas pelo padrão de distribuição, tamanho e forma dos cílios em combinação com a composição química medular. O espécime M.N. Benatti 1400 têm o córtex inferior ao menos em parte mais acentuadamente rugoso e venado, além de mais densamente rizinado, que os espécimes vistos em Benatti (2012a). Nenhum espécime encontrado na área de estudo apresentou picnídios, e todos os apotécios vistos somente apresentaram ascos imaturos (detalhes de ascósporos e conídios podem ser encontrados em Benatti 2012a).

O espécime M.P. Marcelli 11584 é pequeno, com lacínias no tamanho mínimo para a espécie e está bastante danificado e fragmentado (difícil ver reações de coloração, especialmente por conta da oxidação da substância medular; o ácido salazínico foi confirmado em cromatografia, e em baixas quantidades). Devido às características encontradas, entretanto, não há como supor que se trate de outra espécie, mas apenas de um talo mal desenvolvido e danificado.

Parmelinopsis horrescens (Taylor) Elix \& Hale, Mycotaxon 29: 242. 1987 E Parmelia horrescens Taylor, in J.T. Mackay, Flora Hibernica 2: 144. 1836. Tipo: IRELAND. KerRY, Dunkerron Mountains, leg. Taylor (lectótipo, FH-Tayl, n.v.)

Ilustrações de talo em Hale (1976c) e Marcelli (1993); detalhe das estruturas na figura 1 .

Talo cinza esverdeado pardo e escuro em herbário, com até $7,0 \mathrm{~cm}$ diâm. Lacínias de ramificação sub-dicotômica a irregular, 0,5-1,5 mm larg., pouco adnatas, pouco aderidas, contíguas a sobrepostas lateralmente, de ápices truncados a subtruncados, a margem sinuosa a crenada ou subirregular e com linha negra, as axilas ovais a irregulares, a superfície contínua, lisa a subrugosa. Lóbulos ou lacínulas ausentes. Máculas ausentes. Pseudocifelas ausentes. Cílios negros, em geral simples, raramente furcados, $0,10-0,60 \times$ ca. $0,03 \mathrm{~mm}$, não bulbados, frequentes por toda a margem tornando-se abundantes a contíguos nas axilas e crenas das lacínias. Sorédios e pústulas ausentes. Isídios $0,05-0,40 \times$ ca. $0,05 \mathrm{~mm}$, granulares a cilíndricos lisos, simples a parcialmente ramificados, laminais, eretos a raramente procumbentes, concoloridos ou de ápices amarronzados, frequentemente ciliados. Medula branca, sem manchas de pigmentos $\mathrm{K}+$ púrpura. Lado de baixo negro, lustroso, liso a subrugoso, zona marginal marrom a negra, atenuada a indistinta do centro, lustrosa, lisa a papilada, rizinada. Rizinas $0,10-0,70 \times$ ca. $0,03 \mathrm{~mm}$, negras, simples a raramente furcadas ou irregularmente ramificadas, 
homogeneamente distribuídas, abundantes. Apotécios e picnídios não encontrados.

Substâncias de importância taxonômica: atranorina (córtex superior, $\mathrm{K}+$ amarelo, UV-), ácidos 3-metoxi2,4-di-O-metilgirofórico, 5-O-metilhiáscico, algumas substâncias não determinadas ("complexo horrescens"); o espécime encontrado apresenta traços de ácido girofórico, conforme visto em cromatografia (medula, $\mathrm{K}-, \mathrm{C}-, \mathrm{KC}+$ róseo, $\mathrm{P}-, \mathrm{UV}-$ ).

Distribuição conhecida: Oceania, Ásia, Europa, África, América do Norte, América Central e América do Sul. No Brasil é citada para os Estados de GO, MG, PR, RS e SP (Hale 1976c, Swinscow \& Krog 1988 Pereira \& Marcelli 1989, Osorio 1992, Purvis et al. 1992, Marcelli 1993, 2004, Elix 1994b, Esslinger \& Egan 1995, Ribeiro 1998, Eliasaro \& Adler 2000, Eliasaro 2001, Calvelo \& Liberatore 2002, Spielmann 2004, 2005, Divakar \& Upreti 2005).

Material estudado: BRASIL. São PAulo: Município de São Paulo, Serra da Cantareira, Parque Estadual da Cantareira, nas proximidades do Lago das Carpas, 9-I-1991, leg. M.P. Marcelli, A. Rezende \& I. Riquelme 10962 (SP).

Comentários: Somente um espécime de P. horrescens foi encontrado. Esta espécie pode ser facilmente reconhecida pelos isídios ciliados que dão um aspecto de cor escura e suja característico ao talo. Outras características importantes são as substâncias do "complexo horrescens" e traços de ácido girofórico como constituintes medulares, como mencionado por Jungbluth (2006). Pode ser distinguida de P. minarum que não forma cílios nos isídios e apresenta reações $\mathrm{C}+$ e $\mathrm{KC}+$ róseos na medula, devido ao ácido girofórico ser a substância principal.

Comparações dos perfis cromatográficos mostram que as composições químicas de $P$. horrescens e de $P$. subfatiscens são muito semelhantes e diferem bastante do perfil visualizado para espécimes de P. minarum. A diferença de Parmelinopsis subfatiscens para com $P$. horrescens e $P$. minarum é que a espécie apresenta formação de pústulas ciliadas ao invés de isídios ciliados ou eciliados.

O espécime M.N. Benatti 1371 é quase todo composto por $P$. schindleri, mas contém alguns fragmentos com isídios ciliados que aparentemente são de $P$. horrescens, o talo da primeira estava crescendo sobre o desta última. Parmelinopsis schindleri difere pela formação de lacínulas laminais ciliadas ao invés de isídios ou pústulas.
Parmelinopsis minarum (Vainio) Elix \& Hale, Mycotaxon 29: 243. 1987 E Parmelia minarum Vainio. Acta Societatis pro Fauna et Flora Fennica 7: 48. 1890. Tipo: BRASIL. Minas Gerais, Antônio Carlos (Sítio), ad truncos arborum, col. Vainio in Lichenes brasiliensis exsiccati $n^{\circ} 1040$ (holótipo TUR Vainio herbarium n ${ }^{\circ} 2689$ n.v., isótipos: BM, FH, UPS, n.v.).

Ilustrações de talo em Hale (1976c) e Marcelli (1993); detalhe de estruturas na figura 2.

Talo cinza esverdeado pardo em herbário, laciniado, com até $5,5 \mathrm{~cm}$ diâm. Lacínias de ramificação subdicotômica a irregular, 0,5-2,0 mm larg., adnatos, adpressos, contíguas a sobrepostas lateralmente, de ápices truncados a subtruncados, a margem sinuosa a crenada ou subirregular e com linha negra, as axilas ovais a irregulares, a superfície contínua, lisa a ocasionalmente subrugosa. Lacínulas ou lóbulos adventícios ausentes. Máculas ausentes. Pseudocifelas ausentes. Cílios negros, em geral simples, às vezes furcados ou irregularmente ramificados, $0,10-0,50 \times$ ca. $0,03 \mathrm{~mm}$, não bulbados, frequentes por toda a margem tornando-se abundantes a contíguos nas axilas e crenas das lacínias. Pústulas e sorédios ausentes. Isídios 0,05-0,40 × ca. 0,05 mm, cilíndricos lisos, simples a parcialmente ramificados, laminais, eretos a procumbentes, firmes a caducos, concoloridos ou de ápices amarronzados, em geral sem ornamentações ou raramente com cílios. Medula branca a levemente rosada, sem manchas de pigmentos $\mathrm{K}+$ púrpura. Lado de baixo negro, lustroso, liso a rugoso, zona marginal marrom a negra, atenuada ou indistinta do centro, lustrosa, lisa a rugosa, papilada. Rizinas $0,10-0,70 \times$ ca. $0,05 \mathrm{~mm}$, negras, simples a irregularmente ramificadas, frequentes a abundantes, homogeneamente distribuídas. Apotécios (raros) côncavos, 0,5-1,5 mm diâm., sésseis a subestipitados, laminais, margem crenada e isidiada, anfitécio rugoso e isidiado, disco marrom claro, imperfurado, não pruinoso, ascósporos não encontrados (himênio sem ascos). Picnídios não encontrados.

Substâncias de importância taxonômica: atranorina (córtex superior, $\mathrm{K}+$ amarelo, UV-), ácidos girofórico, 5-O-metilhiáscico, 4-O-metilgirofórico (medula, $\mathrm{K}$ - ou + róseo claro, $\mathrm{C}+$ róseo, $\mathrm{KC}+$ róseo, $\mathrm{P}-, \mathrm{UV}-$ ).

Distribuição conhecida: Oceania, Ásia, África, Europa, América do Norte, América Central e América do Sul. No Brasil é citada para os Estados de MG, PR, RS e SP (Zahlbruckner 1930, Osorio 1975, 1992, Hale 1976c, 
Krog \& Swinscow 1979, Swinscow \& Krog 1988, Purvis et al. 1992, Kurokawa 1993, Marcelli 1993, 2004, Elix 1994b, Esslinger \& Egan 1995, Ribeiro 1998, Eliasaro \& Adler 2000, Eliasaro 2001, Kurokawa \& Lai 2001, Calvelo \& Liberatore 2002, Louwhoff \& Elix 2002, Chen et al. 2003, Spielmann 2004, 2005, Canêz 2005).

Material estudado: BRASIL. SÃo PAULO: Município de Mairiporã, Serra da Cantareira, construção de condomínio, 11-III-1989, leg. M.P. Marcelli 6049, 6074, 6076 (SP); idem, Município de São Paulo, Serra da Cantareira, Sítio da Cachoeira, 20-VII-1990, leg. M.P. Marcelli, O. Yano \& A. Rezende \& R.C. Lourenço 9807; idem, Parque Estadual da Cantareira, 9-I-1991, leg. M.P. Marcelli, A. Rezende \& I. Riquelme 10923, 10926, 10948 p. min. p., 10949 (SP); idem, nas proximidades do Lago das Carpas, 9-I-1991, leg. M.P. Marcelli, A. Rezende \& I. Riquelme 10975, 10976, (SP); idem, Núcleo da Pedra Grande, 18-V-1992, leg. M.P. Marcelli, A. Rezende \& O. Yano 13607 (SP); idem, Parque Estadual da Cantareira, próximo ao Lago das Carpas, 25-VI-1991, leg. M.P. Marcelli, O. Yano, A. Rezende \& F.M.M. Coppolla, 11434 (SP); idem, à beira da estrada do Pé de Galinha para Mairiporã, 25-VI-1991, leg. M.P. Marcelli, O. Yano, A. Rezende \& F.M.M. Coppolla, 11581 (SP); VI-2000, leg. M.N. Benatti 1017, 1267, 1302 (SP); idem, Parque Estadual da Cantareira, V-2000, leg. M.M. Benatti 9 (SP).

Comentários: As principais características de Parmelinopsis minarum são as lacínias estreitas e ciliadas, a formação de isídios eciliados e o ácido girofórico como principal constituinte medular. Além desses aspectos, que a distinguem de $P$. horrescens, $P$. minarum foi encontrada fértil na localidade, contudo, apenas um espécime foi encontrado contendo apotécios, todavia com himênios não desenvolvidos e sem ascos. É uma das espécies de pequenas parmélias mais facilmente encontradas no Parque Estadual da Cantareira. Cresce comumente sobre troncos e ramos, mas é frequentemente encontrada também crescendo entrelaçada com diversas espécies de musgos na localidade de estudo.

Tal como mencionado por Canêz (2005) e Jungbluth (2006), alguns dos espécimes encontrados podem ocasionalmente apresentar isídios ciliados similares aos de $P$. horrescens. Entretanto, esses são poucos e os isídios ciliados são escassos em meio aos demais. Análises em cromatografia não evidenciaram perfis distintos desses espécimes para com os demais de P. minarum. Talos de P. horrescens apresentam outro complexo de substâncias, que difere tanto nos testes de coloração (que reagem apenas $\mathrm{KC}+$ róseo) quanto no perfil em cromatografia (referido como "complexo horrescens").

O espécime M.P. Marcelli 11581 tem poucos cílios e rizinas em comparação com os demais, porém nenhuma outra diferença morfológica ou química significativa foi encontrada.

Parmelinopsis schindleri (Hale) Elix \& Hale, Mycotaxon 29: 243. 1987 E Parmelina schindleri Hale, Smithsonian Contributions to Botany 33: 44. 1976. Tipo: BRASIL. Minas Gerais, Caraça, Brazil, Vainio in Lichenes Brasliensis Exsiccati ${ }^{\circ} 284$ (holótipo BM n.v., isótipos FH, M, TUR e UPS n.v.). Ilustrações de talo em Hale (1976c); detalhe de estruturas na figura 3 .

Talo cinza esverdeado pardo em herbário, com até $6,0 \mathrm{~cm}$ diâm. Lacínias de ramificação subdicotômica a irregular, 0,5-1,5(-2,0) mm larg., pouco adnatas a elevadas, pouco aderidas, sobrepostos lateralmente a amontoados, de ápices subtruncados, a margem sinuosa, crenada a subirregular lacinulada e com linha negra, as axilas ovais a irregulares, a superfície contínua, lisa a subrugosa. Lóbulos ou lacínulas frequentes a abundantes, não adventícias, 0,10-1,00 × 0,10-0,80 mm, planas, simples a dicotômica ou irregularmente ramificadas, marginais a laminais prostradas e por vezes sobrepostas, ciliadas a eciliadas. Máculas ausentes. Pseudocifelas ausentes. Cílios negros, em geral simples, raramente furcados, $0,10-0,60 \times$ ca. $0,03 \mathrm{~mm}$, não bulbados, frequentes por toda a margem tornando-se abundantes a contíguos nas axilas e crenas das lacínias. Sorédios, pústulas e isídios ausentes. Medula branca, sem manchas de pigmentos $\mathrm{K}+$ púrpura. Lado de baixo negro, lustroso, liso a subrugoso, zona marginal marrom a negra, atenuada a indistinta do centro, lustrosa, lisa, papilada a rizinada. Rizinas $0,10-0,80 \times$ ca. $0,05 \mathrm{~mm}$, negras, simples a raramente furcadas, homogeneamente distribuídas, frequentes. Apotécios côncavos, 0,5-2,0 mm diâm., sésseis, laminais, margem crenada, anfitécio rugoso, não ornamentados a pouco lacinulados, disco marrom claro, imperfurado, não pruinoso, ascósporos não encontrados (himênio sem ascos ou com ascos imaturos). Picnídios negros, imersos, de ostíolo negro, surgindo em parte das lacínulas em alguns espécimes. Conídios não encontrados.

Substâncias de importância taxonômica: atranorina (córtex superior, $\mathrm{K}+$ amarelo, UV-), ácidos 3-metoxi- 
2,4-di-O-metilgirofórico, 5-O-metilhiáscico, e algumas substâncias indeterminadas ("complexo horrrescens") traços de ácido girofórico (medula, K-, C-, KC- ou + fraco róseo, P-, UV-).

Distribuição conhecida: América do Sul. No Brasil é citada para os Estados de MG, PR, RJ e SP (Hale 1976c, Eliasaro \& Adler 2000, Eliasaro 2001).

Material estudado: BRASIL. SÃo PAulo: Município de São Paulo, Serra da Cantareira, Parque Estadual da Cantareira, 9-I-1991, leg. M.P. Marcelli, A. Rezende \& I. Riquelme 10922, 10924, 10925 (SP); idem, nas proximidades do Lago das Carpas, 9-I-1991, leg. M.P. Marcelli, A. Rezende \& I. Riquelme 10948 p. max. p., 10993, 10994 (SP); idem, próximo ao Lago das Carpas, 25-VI-1991, leg. M.P. Marcelli, O. Yano, A. Rezende \& F.M.M. Coppolla, 11432 (SP); idem, na estrada principal para o Lago das Carpas, 30-III-1992, leg. M.P. Marcelli, A. Rezende \& O. Yano 13408 (SP); idem, Núcleo da Pedra Grande, no bosque, 18-V-1992, leg. M.P. Marcelli, A. Rezende \& O. Yano 13537, 13579 (SP); idem, VI-2000, leg. M.N. Benatti s.n., 3 (SP); idem, nas proximidades do Lago das Carpas, VI-2000, leg. M.N. Benatti 10, (SP); idem, na mata, VI-2000, leg. M.N. Benatti 1018, 1019, 1021, 1064, 1088 (SP); idem, ao lado do museu, VI-2000, leg. M.N. Benatti 1371 p. max. p. (SP).

Comentários: Trata-se de uma das poucas espécies do gênero a apresentar reprodução indireta sob a forma de lóbulos, semelhante à $P$. spathulata que difere pela formação de isídios e lóbulos principalmente laminais, e pela composição química medular parcialmente distinta (contendo os ácidos 5-O-metilhiáscico e 3-metil-2,4-O-metilgirofórico, mais o ácido 4-O-metilgirofórico e sem ácido girofórico, segundo Jungbluth 2006).

A densa produção de lacínulas associada à química medular (substâncias do "complexo horrescens" e traços de ácido girofórico) faz com que $P$. schindleri seja de fácil identificação, até mesmo em campo na área de estudo, onde é facilmente encontrada sobre cascas de árvore. Dependendo da concentração de substâncias a reação pode variar de tonalidade, ora rosa mais claro, ora rosa mais distinto.

Existe bem pouca bibliografia sobre esta espécie, incluindo apenas algumas poucas descrições. Há espécimes que apresentam lacínulas mais estreitas, outros geralmente eciliadas ou ocasionalmente com raros cílios, enquanto que alguns podem ou não apresentar picnídios nas lacínulas, podendo ser variedades incomuns ou representarem um táxon similar ainda não descrito. Há vários talos aparentemente intermediários, o que dificulta saber qual representaria uma variação morfológica de que táxon, ainda mais porque testes de coloração medulares não evidenciam nenhuma reação positiva em alguns espécimes, nem mesmo $\mathrm{KC}+$ róseo, embora os perfis cromatográficos aparentem ser semelhantes (se houver mais de uma espécie envolvida, estão ligadas ao "complexo horrescens").

Alguns poucos espécimes apresentam formação de isídios que se convertem em lacínulas de mesmo aspecto dos demais espécimes de $P$. schindleri. Segundo Jungbluth (2006) estes podem tratar-se talvez de $P$. spathulata. Estes espécimes variam quanto a quantidade de isídios e o quão rápido estes começam a adquirir o aspecto de lacínulas, mas possuem química similar aos demais vista em CCD. Estudos com os materiais tipos de $P$. schindleri, de $P$. spathulata e seus sinônimos são necessários para elucidar essas questões.

Assim como P. minarum, P. schindleri é bastante comum e também é encontrada frequentemente entrelaçada com briófitas na localidade de estudo. Parmelinopsis schindleri normalmente apresenta lacínulas principalmente marginais, não originadas de isídios, não picnidiadas, e ciliadas, diferindo de P. spathulata pelas lacínulas originárias em meio aos isídios laminais, e sempre eciliadas.

Parmelinopsis subfatiscens (Kurokawa) Hale, Mycotaxon 29: 243. 1987 P Parmelia subfatiscens Kurokawa, in Hale \& Kurokawa, Contributions from the United States National Herbarium 36: 134. 1964. Tipo: ÁFRICA DO SUL. TRANSVAAL, Zoutpansberg, Louis Trichardt, leg. Alborn 6443 (holótipo LD, n.v.). Ilustrações de talo em Hale (1976c); detalhe de estruturas na figura 4 .

Talo cinza esverdeado pardo claro, com até $3,0 \mathrm{~cm}$ diâm. Lacínias de ramificação subdicotômica a irregular, 0,5-1,0 mm larg., adnatas, aderidas, contíguas a pouco sobrepostas lateralmente, de ápices subtruncados, a margem sinuosa, lisa a crenada ou subirregular sem linha negra, as axilas ovais a irregulares, a superfície contínua, lisa a subrugosa. Lóbulos ou lacínulas ausentes a escassas, $0,2-0,8 \times 0,1-0,4 \mathrm{~mm}$, laminais, simples de formato irregular, ciliadas, surgindo em meio às pústulas. Máculas ausentes. Pseudocifelas ausentes. Cílios negros, em geral simples ou às vezes irregularmente ramificados, $0,10-0,30(-0,60) \times$ ca. $0,03 \mathrm{~mm}$, não bulbados, frequentes por toda a margem tornando-se 

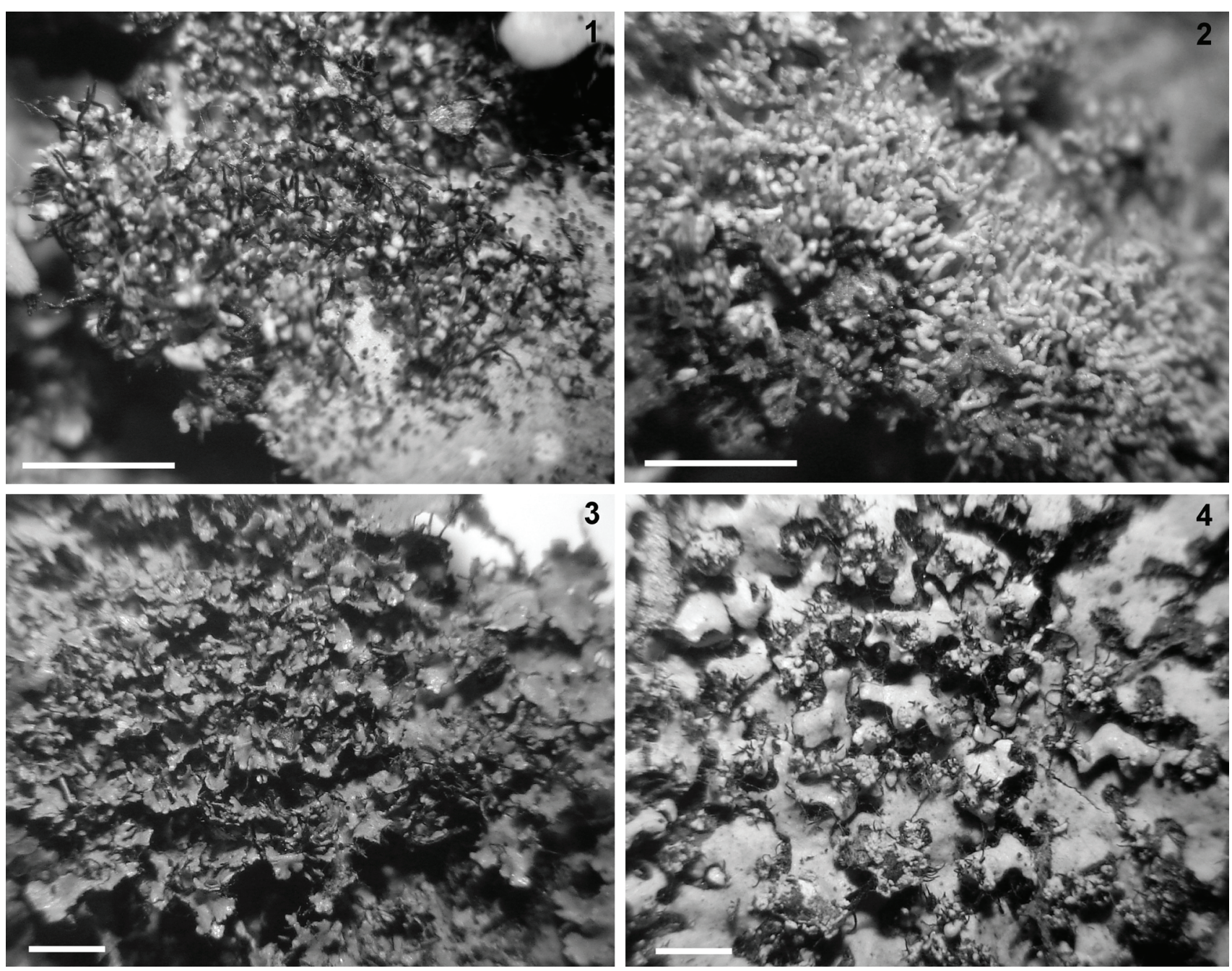

Figuras 1-4. Detalhes das estruturas em espécimes de Parmelinopsis no Parque Estadual da Cantareira, São Paulo, SP, Brasil. 1. Isídios ciliados de Parmelinopsis horrescens (M.P. Marcelli 10962). 2. Isídios eciliados de Parmelinopsis minarum (M.P. Marcelli 10975). 3. Lacínulas de Parmelinopsis schindleri (M.P. Marcelli 10922). 4. Pústulas ciliadas de Parmelinopsis subfatiscens (M.P. Marcelli 13602). Barra $=1 \mathrm{~mm}$.

Figures 1-4. Details of the structures of Parmelinopsis specimens in the Parque Estadual da Cantareira, São Paulo, São Paulo State, Brazil. 1. Ciliate isidia of Parmelinopsis horrescens (M.P. Marcelli 10962). 2. Eciliate isidia of Parmelinopsis minarum (M.P. Marcelli 10975). 3. Lacinulae in Parmelinopsis schindleri (M.P. Marcelli 10922). 4. Ciliate pustulae of Parmelinopsis subfatiscens (M.P. Marcelli 13602). Bars $=1 \mathrm{~mm}$.

abundantes a contíguos nas axilas e crenas das lacínias. Sorédios e isídios ausentes. Pústulas em forma de rugas irregulares ou às vezes de aspecto isidióide, parcialmente rebentando e se decompondo em grânulos grosseiros expondo a medula, submarginais a laminais principalmente sobre dobras do talo, ciliadas. Medula branca, sem manchas de pigmentos $\mathrm{K}+$ púrpura. Lado de baixo negro, lustroso, liso a subrugoso, zona marginal marrom a negra, atenuada a indistinta do centro, lustrosa, lisa a pouco venada, papilada ou rizinada. Rizinas $0,20-0,60 \times$ ca. $0,05 \mathrm{~mm}$, negras, simples ou às vezes irregularmente ramificadas, homogeneamente distribuídas, frequentes a abundantes. Apotécios e picnídios não encontrados.

Substâncias de importância taxonômica: atranorina (córtex superior, $\mathrm{K}+$ amarelo, UV-), ácidos 3-metoxi-2,4di-O-metilgiroforicolecanórico, 5-O-metilhiáscico, algumas substâncias indeterminadas ("complexo horrescens"), traços de ácido girofórico (medula, K-, $\mathrm{C}$ - ou + fraco róseo, $\mathrm{KC}+$ róseo, $\mathrm{P}-, \mathrm{UV}-$ ).

Distribuição conhecida: Oceania, África, América do Norte, América Central e América do Sul. No Brasil é citada para o Estado de SP (Hale 1976c, Swinscow \& Krog 1988, Elix 1994b, Ribeiro 1998, Brodo et al. 
2001, Calvelo \& Liberatore 2002, Jungbluth 2006, Marcelli 2004).

Material estudado: BRASIL. SÃo PAULo: Município de São Paulo, Serra da Cantareira, Parque Estadual da Cantareira, Núcleo da Pedra Grande, no bosque, 18-V1992, leg. M.P. Marcelli, A. Rezende \& O. Yano 13542 (SP); idem, próximo ao Lago das Carpas, VI-1992, leg. M.N. Benatti 1266 (SP); idem, na mata, VI-1992, leg. M.N. Benatti 1270, 1281 (SP).

Comentários: Parmelinopsis subfatiscens tem como característica principal a formação de pústulas ciliadas que ocasionalmente rompem expondo uma área enegrecida, o córtex inferior, pela perda das hifas da medula, além da presença de substâncias medulares do "complexo horrescens".

Esta característica de pústulas, que é bastante incomum em parmélias, levantou dúvidas sobre a identificação, mas a bibliografia demonstra que Hale (1976c) havia encontrado a espécie no Caribe e na África do Sul. O material estudado aqui está mais de acordo com os espécimes sul-africanos, pela presença de cílios que Hale (1976c) mencionou não ter encontrado em seus espécimes do continente americano (ele pode ter encontrado espécimes similares de $P$. spumosa).

Os testes químicos e a cromatografia confirmaram a presença do "complexo horrescens", também observadas por Hale (1976c), e devido ao grau de compatibilidade morfológica e química pode-se supor que se trata do provável par específico de $P$. horrescens, uma vez que não é ainda conhecida uma espécie sorediada com essa composição química medular.

Existe muita confusão em literatura envolvendo duas espécies pustuladas de Parmelinopsis: $P$. subfatiscens e $P$. spumosa. O material encontrado na localidade de estudo está em acordo com a descrição original de $P$. subfatiscens, e a análise cromatográfica revela perfis muito similares aos de $P$. horrescens, enquanto que por comparação, perfis de espécimes de P. spumosa (comparados com materiais do herbário SP oriundos de outras localidades), que forma pústulas sem cílios e de medula levemente amarelada, apresentam perfis similares aos de P. minarum.

\section{Agradecimentos}

O autor agradece a FAPESP (Fundação para o Amparo à Pesquisa do Estado de São Paulo) pela concessão da Bolsa de Iniciação Científica $n^{\circ}$ 00/01009-1.

\section{Literatura citada}

Aptroot, A. 1990. Lichens of Madagascar: new and interesting records and species. Cryptogamie, Bryologie et Lichenologie 11: 401-408.

Asahina, Y. \& Shibata, S. 1954. Chemistry of Lichen Substances. Japan Society for the Promotion of Science. Tóquio.

Awasthi, D.D. 1976. Lichen genus Parmelia in India I - Subgenera Parmelia and Amphigymnia. Biological Memoirs 1: 155-229.

Benatti, M.N. 2010. Revisão taxonômica do gênero Bulbothrix Hale (Parmeliaceae, Ascomycota liquenizados). Tese de Doutorado, Instituto de Botânica, São Paulo.

Benatti, M.N. 2012a. Canoparmelia cinerascens belongs in the genus Parmelinella (Parmeliaceae, lichenized Ascomycota). Opuscula Philolichenum 11: 26-30.

Benatti, M.N. 2012b. A review of the genus Bulbothrix Hale: the species with medullar norstictic or protocetraic acids. MycoKeys 2: 1-28.

Benatti, M.N. \& Marcelli, M.P. 2007. Gêneros de fungos liquenizados dos manguezais do Sul-Sudeste do Brasil, com enfoque no manguezal do Rio Itanhaém, Estado de São Paulo. Acta Botanica Brasilica 21: 863-878.

Brako, L., M.J. Dibben, \& Amaral, I. 1985. Preliminary notes on the macrolichens of Serra do Cachimbo, northcentral Brazil. Acta Amazonica, suplemento ("Contribuições do Projeto Flora Amazônica". Parte 2) 15: 123-135.

Brodo, I.M., Sharnoff, S.D. \& Sharnoff, S. 2001. Lichens of North America. Yale University Press, New Haven.

Bungartz, F. 2001. Analysis of lichen substances. Em http:// ces.asu.edu/ASULichens/plb 400/laboratory/ chemistry/ tlc.html (acesso em 01.10.2004).

Calvelo, S. \& Liberatore, S. 2002. Catálogo de los Líquenes de la Argentina. Kurtziana 29: 7-170.

Canêz, L.S. 2005. A família Parmeliaceae na localidade de Fazenda da Estrela, município de Vacaria, Rio Grande do Sul, Brasil. Dissertação de Mestrado. Instituto de Botânica, São Paulo.

Chen, B.J., Xu, L., Qian, Z.G., Elix, J.A. 2003. Parmeliaceae (Ascomycota) lichens in China's Mainland. I. The genus Hypotrachyna. Mycotaxon 86: 359-373.

Divakar, P.K. \& Upreti, D.K. 2005. Parmelioid Lichens in India (A Revisionary Study). Bishen Singh Mahendra Pal Singh, Dehra Dun.

Dodge, C.W. 1959. Some lichens of tropical Africa III. Parmeliaceae. Annals of the Missouri Botanical Garden 46: 39-193.

Eliasaro, S. 2001. Estudio taxonómico y florístico sobre las Parmeliaceae sensu stricto (Ascomycota Liquenizados) del Segundo Planalto del Estado de Paraná, Brasil. Tese de Doutorado, Universidad de Buenos Aires. 
Eliasaro, S. \& Adler, M.T. 1997. Two new species and new reports in the Parmeliaceae sensu stricto (lichenized Ascomycotina) from Brazil. Mycotaxon 63: 49-55.

Eliasaro, S. \& Adler, M.T. 2000. The species of Canomaculina, Myelochroa, Parmelinella, and Parmelinopsis (Parmeliaceae, lichenized Ascomycotina) from the Segundo Planalto in the state of Paraná, Brazil. Acta Botanica Brasilica 14: 141-149.

Elix, J.A. 1994a. Bulbothrix. In: Orchard, A.E. \& Grgurinovic, C. (eds.). Flora of Australia, Lichens. Introduction, Lecanorales 2. v.55. Australia Government Publishing Service, Canberra, pp. 13-19.

Elix, J.A. 1994b. Parmelinopsis. In: Orchard, A.E. \& Grgurinovic, C. (eds.). Flora of Australia, Lichens. Introduction, Lecanorales 2. v.55. Australia Government Publishing Service, Canberra, pp. 131-138.

Esslinger, T.L. \& Egan, R.S. 1995. A sixth checklist of the lichen-forming, lichenicolous, and allied fungi of the continental states and Canada. The Bryologist 98: 467-549.

Feuerer, T.E. (ed.) 2008. Checklists of lichens and lichenicolous fungi. Versão 1 Setembro de 2008. In http://www.checklists.de (acesso em 10.2008).

Fink, B. 1905. How to collect and study lichens. The Bryologist 8: 22-27.

Galloway, D.J. 1985. Flora of New Zealand - lichens. Wellington, Government Printer.

Galloway, D.J. 2007. Flora of New Zealand Lichens. Revised Second Edition Including Lichen-Forming and Lichenicolous Fungi. Volumes 1 and 2. Manaaki Whenua Press, Lincoln, New Zealand.

Galloway, D.J. \& Quilhot, W. 1998. Checklist of Chilean lichen-forming and lichenicolous fungi. Gayana Botanica 55: 111-185.

Gumboski, E.L. \& Eliasaro, S. 2011. Checklist of lichenized fungi of Santa Catarina State (Brazil). Mycotaxon 115: 535.

Hale, M.E. 1976a. A Monograph of the Lichen Genus Pseudoparmelia Lynge (Parmeliaceae). Smithsonian Contributions to Botany 31: 1-62.

Hale, M.E. 1976b. A Monograph of the Lichen Genus Bulbothrix Hale (Parmeliaceae). Smithsonian Contributions to Botany 32: 1-29.

Hale, M.E. 1976c. A monograph of the lichen genus Parmelina Hale (Parmeliaceae). Smithsonian Contributions to Botany 33: 1-60.

Hale, M.E. 1979. How to know the Lichens. The PicturedKey Nature Series. WM.C. Brown Company Publishers. Dubuque.

Hale, M.E. 1987. How to Know the Lichens. $2^{\text {nd }}$ ed. WCB/ McGraw-Hill. Boston.

Hale, M.E. \& Kurokawa, S. 1964. Studies on Parmelia subgenus Parmelia. Contributions from the United States National Herbarium 36: 121-191.
Huneck, S. \& Yoshimura, I. 1996. Identification of Lichen Substances. Springer, Berlin.

Jungbluth, P. 2006. A família Parmeliaceae (fungos liquenizados) em fragmentos de cerrados do Estado de São Paulo. Dissertação de Mestrado, Instituto de Botânica, São Paulo.

Junghuhn, F. 1855. Plantae Junghuhnianae. Enumeratio plantarum, quas in Insulis Java et Sumatra detexit. Fasc. IV, Lugduni Batavorum, 1851-1855: 427-494.

Krog, H. \& Swinscow, T.D.V. 1979. The Parmelia subgenus Hypotrachyna in East Africa. Norwegian Journal of Botany 26: 11-43.

Kurokawa, S. 1993. Nepalese genera and species of the Parmeliaceae with notes on three additional and one rare species. Annals of the Tsukuba Botanical Garden 12: $75-81$.

Kurokawa, S. \& Lai, M.J. 2001. Parmelioid lichen genera and species in Taiwan. Mycotaxon 77: 225-284.

Lopez-Figueiras, M. 1986. Censo de macroliquenes venezolanos de los estados Falcon, Lara, Merida, Tachira y Trujillo. Facultad de Farmacia, Universidad de Los Andes, Merida.

Louwhoff, S.H.J.J. \& Elix, J.A. 2000. The lichens of Rarotonga, Cook Islands, South Pacific Ocean II: Parmeliaceae. Lichenologist 32(1): 49-55.

Louwhoff, S.H.J.J. \& Elix, J.A. 2002. The Parmeliaceae (lichenized Ascomycota) of New Caledonia. Lichenologist 34: 373-394.

Lynge, B. 1914. Die Flechten der ersten Regnellschen Expedition. Die Gattungen Pseudoparmelia gen. nov. und Parmelia Ach. Arkiv för Botanik 13: 1-172.

Malcolm, W.M. \& Galloway, D.J. 1997. New Zeland Lichens: Checklist, Key and Glossary. Museum of New Zeland, Te Papa Tongarewa.

Marcano, V., Morales-Méndez, A., Sipman, H. \& Calderon, L. 1996. A first checklist of the lichenforming fungi of the Venezuelan Andes. Tropical Bryology 12: 193-235.

Marcelli, M.P. 1990. Liquens de restingas e manguezais da ilha do Cardoso. Anais do II Simpósio de Ecossistemas da Costa Sul e Sudeste Brasileira (Águas de Lindóia, SP). v.3: 382-392.

Marcelli, M.P. 1991. Aspects of the foliose lichen flora of the southern-central coast of São Paulo State, Brazil. In: D.J. Galloway (ed.). Tropical Lichens: Their Systematics, Conservation, and Ecology, Systematics Association, Special Volume 43, Clarendon Press, Oxford, pp. 151-170

Marcelli, M.P. 1993. Pequenas Parmelia s.l. Ciliadas dos Cerrados Brasileiros. Acta Botanica Brasilica 7: 25-70.

Marcelli, M.P. 1998a. History and current knowledge of Brazilian Lichenology. In: M.P. Marcelli, \& M.R.D. Seaward (eds.). Lichenology in Latin America: history, current knowledge and applications. CETESB, São Paulo, pp. 25-45. 
Marcelli, M.P. 1998b. Diversidade dos fungos liquenizados no Estado de São Paulo: um diagnóstico. In: C.A. Joly, \& C.E.M. Bicudo (eds.). Biodiversidade do Estado de São Paulo, Brasil: síntese do conhecimento ao final do século XX. v.2. FAPESP, São Paulo, pp. 25-35

Marcelli, M.P. 2004. Checklist of lichens and lichenicolous fungi of Brazil. Version 1: June 2004. http://www. biologie.uni-hamburg.de/checklists/brazil_1.htm (acesso em 10.2004).

Montagne, J.F.C. 1856. Sylloge generum specierumque cryptogamarum, quas in variis operibus descriptas iconibusque illustratas, nunc ad diagnosim reductas, nonnullasque novas interjectas, ordine systematico disposuit. Paris.

Orange,A., James, P.W. \& White, F.J. 2001. Microchemical methods for the identification of lichens. The British Lichen Society, London.

Osorio, H.S. 1975. Contribution to the Lichen Flora of Uruguay. VIII. Additions and corrections. Comunicaciones Botánicas del Museo de Historia Natural de Montevideo 4: 1-12.

Osorio, H.S. 1989. Contribution to the Lichen Flora of Brazil. XXIII. Lichens from São Paulo City. Mycotaxon 36: 161-162.

Osorio, H.S. 1992. Contribucion a la flora liquénica del Uruguay. XXV. Líquens publicados entre 1972 a 1991. Anales del Museo Nacional de Historia Natural de Montevideo (Series 2) 8: 43-70.

Papong, K., Boonpragob, K. \& Lücking R. 2007. New species and new records of foliicolous lichens from Thailand. Lichenologist 39: 47-56.

Pereira, W.R. \& Marcelli, M.P. 1989. Liquens da Reserva Biológica do Alto da Serra de Paranapiacaba. Acta Botanica Brasilica 3: 89-94.

Purvis, O.W., Coppins, B.J., Hawksworth, D.L., James, P.W. \& Moore, D.M. 1992. The lichen flora of Great Britain and Ireland. Natural History Museum, London.
Ribeiro, C.H. 1998. A família Parmeliaceae (Ascomycota liquenizados) em Regiões montanhosas dos Estados de Minas Gerais, Rio de Janeiro e São Paulo. Dissertação de Mestrado, Universidade de São Paulo, São Paulo.

São Paulo. 1988. Vegetação Significativa do Município de São Paulo. Secretaria do Meio Ambiente (SMA). Secretaria Municipal de Planejamento (SEMPLA), São Paulo.

Sipman, H.J.M. \& Wolf, J.H.D. 1998. Provisional checklist for the lichens of Chiapas. Acta Botanica Mexicana 45: 1-29.

Soares, D. (coord.) 1999. Paisagem Paulista: Áreas Protegidas. Empresa das Artes, São Paulo.

Spielmann, A.A. 2005. A família Parmeliaceae (fungos liquenizados) nos barrancos e peraus da encosta da Serra Geral, no Vale do Rio Pardo, Rio Grande do Sul, Brasil. Dissertação de Mestrado, Instituto de Botânica, São Paulo.

Spielmann, A.A. 2006. Checklist of lichens and lichenicolous fungi of Rio Grande do Sul (Brazil). Caderno de Pesquisa. Série Biologia (UNISC) 18: 7-125.

Swisncow, T.D.V. \& Krog, H. 1988. Macrolichens of East Africa. British Museum of Natural History. London.

Vainio, E.A. 1915. Additamentum ad lichenographiam Antillarum illustrandam. Annales Academiae Scientiarum Fennicae A6, 7: 1-226.

Walker, J.W. \& James, P.W. 1980. A revised guide to microchemical techniques for the identification of lichen products. British Lichen Society Bulletin 46 (supplement): 13-29.

White, F.J. \& James, P.W. 1985. A new guide to microchemical techniques for the identification of lichen substances. British Lichen Society Bulletin 57 (supplement): 1-41.

Zahlbruckner,A. 1928. Neue und ungenügend beschriebene javanische Flechten. Annales de Cryptogamie Exotique 1: 109-212.

Zahlbruckner, A. 1930. Catalogus lichenum universalis. Vol. VI. Gebrüder Borntraeger, Leipzig. 